\title{
Actitudes de estudiantes de una universidad pública sobre la aplicación del aprendizaje invertido
}

\author{
Attitudes of Students From a Public University About the Application of the Flipped \\ Learning
}

\section{Atitudes de estudantes de uma universidade pública sobre a aplicação da aprendizagem invertida}

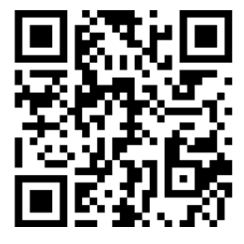

\author{
Edith Chambi-Mescco \\ Universidad Nacional Mayor de San Marcos \\ Lima, Perú \\ echambim@unmsm.edu.pe \\ https://orcid.org/0000-0002-0535-5906
}

Recibido • Received • Recebido: 24 / 08 / 2019
Corregido • Revised • Revisado: 19 / 05 / 2021
Aceptado • Accepted • Aprovado: 07 / 06 / 2021

Resumen: El enfoque denominado aprendizaje invertido ha estado llamando la atención del personal docente en los últimos años, debido a su novedosa forma de programar y ejecutar las sesiones de aprendizaje, lo que ha marcado una diferencia con el modelo tradicional. Se ha aplicado este enfoque en diversos programas de educación superior, los resultados expresan los logros que se han obtenido en términos de aprendizaje. El objetivo del presente artículo de investigación es identificar las actitudes que tiene el estudiantado de segundo ciclo de estudios generales de una universidad pública peruana, luego de haber experimentado el aprendizaje invertido en la asignatura de Lenguaje y Comunicación, en el semestre académico 2018-II. Este estudio es cuasiexperimental y de enfoque mixto. Se aplicó un cuestionario, elaborado por Farrah y Qawasmeh (2018), para recoger las actitudes de 70 estudiantes de dos aulas y que llevaron el curso dirigido por el mismo docente. Los resultados indican que el estudiantado considera que el enfoque pedagógico aprendizaje invertido es altamente beneficioso para sus aprendizajes y sugieren que su eficacia depende de la autonomía que tenga el estudiantado para desarrollar las actividades previas y de las facilidades que tengan para acceder a internet.

Palabras claves: Actitudes; aprendizaje invertido; educación superior; enfoque pedagógico. 
http://doi.org/10.15359/ree.25-3.5

http://www.una.ac.cr/educare

educare@una.ac.cr

\begin{abstract}
The approach called flipped learning has been attracting teachers' attention in recent years due to its novel way of programming and executing learning sessions, thus making a difference with the traditional model. This approach has been applied in various higher education programs; the results express the achievements that have been obtained in terms of learning thanks to its implementation. The purpose of the present research article is to identify students' attitudes from the second cycle of General Studies of a Peruvian public university after having experienced the flipped learning in the course Language and Communication in the academic semester 2018-II. This study is quasi-experimental and of a mixed approach. A questionnaire prepared by Farrah y Qawasmeh (2018) was applied to collect the attitudes of 70 students grouped into two classrooms and developed the subject directed by the same teacher. The results show that most students consider that the pedagogical approach called flipped learning is highly beneficial for their learning because it promotes developing the necessary skills for their professional training. The results also suggest that its effectiveness depends on the students' autonomy to develop the previous activities and the easy access they could have to the Internet.
\end{abstract}

Keywords: Attitudes, flipped learning, higher education, pedagogical approach.

Resumo: $O$ enfoque denominado aprendizagem invertida tem chamado a atenção do corpo docente nos últimos anos, devido a nova maneira de planejar e realizar as sessões de aprendizagem diferenciando-se do modelo tradicional. Este enfoque tem sido aplicado em diversos programas de educação superior, os resultados demostram as conquistas que se tem conseguido em termos de aprendizagem graças a sua implementação. O objetivo do presente artigo de investigação é identificar as atitudes que tem os estudantes do segundo período acadêmico de Estudos Gerais de uma universidade pública peruana, logo após ter experimentado a aprendizagem invertida na disciplina de Linguagem e Comunicação no semestre acadêmico de 2018-II. Este estudo é quaseexperimental e de abordagem mista. Foi aplicado um questionário, elaborado por Farrah y Qawasmeh (2018), para coletar os dados referentes as atitudes de 70 estudantes divididos em duas salas de aulas as quais tiveram o curso ministrado pelo mesmo docente. Os resultados indicam que grande parte desses estudantes consideram que o enfoque pedagógico de aprendizagem invertida é altamente benéfico para o seu processo de aprendizagem, pois promove o desenvolvimento das habilidades necessárias na sua formação profissional e sugerem principalmente que sua eficácia depende da autonomia que tenha como estudante para desenvolver as atividades prévias e das facilidades que tenham para acessar a internet.

Palavras-chave: Atitudes; aprendizagem invertida; educação superior; enfoque pedagógico.

\title{
Introducción
}

En el contexto actual, se han observado diversas propuestas, en términos de metodologías, para el logro y mejora de los aprendizajes. Esta es una preocupación de diversos agentes que intervienen en el proceso educativo, principalmente del personal docente, quienes con base en las necesidades de la realidad educativa en la que se desenvuelven, han propuesto formas de hacer frente a la situación. Tal es el caso de los profesores Jonathan Bergmann y Aaron Sams, 
http://doi.org/10.15359/ree.25-3.5

quienes, en el año 2007, idearon y socializaron una forma de gestionar mejor el tiempo de la clase presencial en una escuela de educación secundaria en Colorado (EE.UU.). Esto surgió ante la necesidad del estudiantado de tener su docente cerca cuando deseaban soporte para desarrollar las actividades de mayor complejidad: "el momento en que los alumnos necesitan que esté físicamente presente con ellos es cuando se atascan en un tema y necesitan mi ayuda personal. No me necesitan en el aula con ellos para darles contenidos; los contenidos los pueden recibir por su cuenta" (Bergmann y Sams, 2014, p. 18).

Efectivamente, el estudiantado no requiere del personal docente solo para que le exponga información sobre un tema en particular, sino que necesita una persona docente que monitoree e intervenga durante las actividades auténticas. Esta forma de llevar la sesión fue la que se denominó clase invertida o flipped classroom (Bergmann y Sams, 2014).

En razón de las ventajas de aplicar enfoques innovadores en el aula para favorecer los aprendizajes, tal como el aprendizaje invertido, se requieren estudios que permitan conocer cómo percibe el estudiantado su experiencia con esta forma de aprender. Por tal motivo, el presente estudio tiene como objetivo identificar las actitudes del estudiantado de una universidad pública peruana acerca del aprendizaje invertido, luego de haber experimentado este enfoque en la asignatura de Lenguaje y Comunicación correspondiente al semestre académico 2018-II. El detalle del estudio se explicará en los siguientes apartados.

\section{Estado de la cuestión}

Se han realizado diversos estudios acerca de la aplicación de este enfoque en educación superior. El objetivo ha sido identificar los logros de esta estrategia, en términos de aprendizaje, en asignaturas vinculadas a las ciencias, al aprendizaje de idiomas, entre otros. La mayoría de los estudios revisados (80\%) se realizaron en el nivel de educación superior y en estos se concluyen que este enfoque promueve mejoras en el aprendizaje del estudiantado (Akçayır y Akçayır, 2018). Asimismo, ha surgido en los últimos años una preocupación por la actitud del estudiantado con base en la experiencia que han tenido de este enfoque en el aula (Sánchez Vera et al., 2016). Se evidencia, entonces, una necesidad de conocer cómo responde el estudiantado al enfoque.

Sobre la eficacia de este enfoque en las aulas, López y Flores Herrera (2013) realizaron un estudio con el objetivo de mejorar el rendimiento del estudiantado en la asignatura de Física en una universidad ecuatoriana, lo hicieron empleando el modelo pedagógico clase invertida. Para tal fin, se diseñaron videos con el contenido del curso; previamente se aplicó una prueba de entrada, luego se evaluó al estudiantado sobre los contenidos de los videos, se trabajaron los problemas planteados en clase y, finalmente, se tomó una prueba de salida. La hipótesis del estudio fue "la media de las diferencias del rendimiento de los estudiantes en la unidad de Hidrostática aplicando la clase invertida es mayor" (López y Flores Herrera, 2013, 
http://doi.org/10.15359/ree.25-3.5

http://www.una.ac.cr/educare

educare@una.ac.cr

p. 79). Los resultados de este estudio indican que se mejoró notablemente el rendimiento del estudiantado, pues la media de sus calificaciones fue de 4,25 en la prueba de entrada, mientras que en la prueba de salida obtuvieron un 8,445; además "la prueba t emparejada aplicada entre la prueba de salida y prueba de entrada dio un valor de $\mathrm{t}=36,9395$ y df=39 con un nivel de significación $\mathrm{p}<0,0001$. Por lo tanto, se acepta la hipótesis de investigación" (López y Flores Herrera, 2013, p. 80). La limitación de este estudio, como los mismos autores señalan, es la carencia de una comparación con grupo de control, por lo que se requieren más estudios que consideren este requisito, de modo que puedan compararse los resultados y observar si hubo logros de aprendizaje o no.

Melo y Sánchez (2017) se propusieron como objetivo obtener las percepciones del estudiantado sobre el empleo del enfoque aprendizaje invertido en su aprendizaje de la asignatura Técnicas avanzadas en laboratorios de análisis de residuos de medicamentos veterinarios y contaminantes. Para ello se empleó un cuestionario validado por cinco personas investigadoras en Didáctica de las ciencias, la prueba de alfa de Cronbach arrojó un coeficiente de fiabilidad de 0,837 para los diez ítems, por lo que la fiabilidad del instrumento es moderadamente alta. Los resultados que se obtuvieron advierten que "Ios participantes creen que su aprendizaje fue más activo y experiencial frente a otras experiencias con metodologías expositivas" (Melo y Sánchez, 2017, p. 33). El estudio expone de forma clara que el estudiantado valoró de forma positiva el enfoque.

En esa misma línea, Farrah y Qawasmeh (2018) realizaron un estudio denominado Actitudes de los estudiantes de inglés hacia el uso de la clase invertida en el aprendizaje de idiomas en la Universidad de Hebron. Se aplicó un cuestionario que contenía una escala de Likert para identificar las actitudes del estudiantado del curso de inglés hacia la estrategia de la clase invertida. El método empleado fue cuantitativo y cualitativo, los resultados indican que la aplicación de esta estrategia promueve el aprendizaje autónomo y la autodirección.

Fauzan y Novary Ngabut (2018), en su investigación sobre la percepción del estudiantado acerca de la implementación de la clase invertida en las clases de escritura, indican que se obtuvieron resultados positivos en la evaluación de los aprendizajes del estudiantado luego de la aplicación del enfoque flipped learning, sus trabajos mejoraron gracias también al papel del personal docente, quien, desde este enfoque, brindaba retroalimentación a cada uno de los trabajos; además, el estudiantado mejoró en su compromiso con la asignatura y revisaron, fuera de aula y más a fondo, los materiales proporcionados por el personal docente.

En los estudios de revisión bibliográfica de este enfoque se verifica que cuando se aplica de forma efectiva, el estudiantado se compromete a aprender, por ello se requiere el profesionalismo del personal docente para poder trabajar en línea, proporcionar comentarios sobre los trabajos del estudiantado y calificarlos a tiempo (Fauzan y Novary Ngabut, 2018). 
http://doi.org/10.15359/ree.25-3.5

También, la mayoría de los desafíos del aprendizaje invertido está relacionada con actividades fuera de la clase: la preparación inadecuada del estudiantado, la necesidad del estudiantado de una orientación antes de la clase presencial (Akçayır y Akçayır, 2018). El aprendizaje invertido plantea estos desafíos que deben ser considerados por el personal docente, de modo que planifique y ejecute las estrategias más apropiadas que le permitan monitorear de forma eficaz el trabajo preparatorio que se da fuera del aula.

El número de estudios sobre este enfoque ha aumentado cada año, dado que sigue creciendo en popularidad (Akçayır y Akçayır, 2018), la mayoría muestra resultados luego de su aplicación en el aula. En definitiva, se identifica como ventaja, en estos estudios, que el enfoque aprendizaje invertido mejora la motivación hacia el aprendizaje promoviendo actitudes positivas en el estudiantado (Akçayır y Akçayır, 2018), al tener la posibilidad de aplicar la información y crear nuevos conocimientos (Awidi y Paynter, 2019). Además, el estudiantado concibe que el tiempo es empleado de forma más eficaz con el aprendizaje invertido (Sánchez Cruzado, 2017), valoran en sí los beneficios para el aprendizaje que promueve este enfoque. Los estudios también reconocen que existen algunos desafíos en torno a la aplicación eficaz de este enfoque, desafíos que motivan nuevas líneas de investigación para los sujetos interesados en el tema.

\section{Marco teórico}

El enfoque o modelo pedagógico aprendizaje invertido supone invertir la enseñanza tradicional al trasladar la instrucción directa fuera de la clase (Tourón et al., 2014), pues los procesos pedagógicos que implican recibir información, los que tradicionalmente se incluían en el aula, tienen lugar fuera de ella gracias a los recursos que hoy en día ofrece la tecnología. $Y$ es que, actualmente, se cuenta con los denominados "e-estudiantes", quienes son "personas que dentro y fuera de las aulas emplean las nuevas tecnologías como herramientas [para su] aprendizaje" (Berenguer Albaladejo, 2016, p. 1466), característica que se aprovecha bajo este enfoque para promover que el estudiantado revise información previamente a la clase presencial. En suma, consiste fundamentalmente en emplear videos, presentaciones, audios, lecturas, entre otros, como recursos para que sean revisados por el estudiantado fuera de aula; mientras que dentro se desarrollan actividades, dinámicas y otras estrategias que facilitan participación e interacción entre docente-estudiante y estudiante-estudiante (Martín Rodríguez y Santiago Campión, 2016); lo cual significa una renovación de la clase presencial gracias al uso de las tecnologías (Sánchez Vera et al., 2016).

Entre las ventajas de este enfoque se encuentran, por ejemplo, la posibilidad que tiene el estudiantado de aprender a su propio ritmo, pues se les brinda cierta flexibilidad para acceder a los recursos, debido a que se comparten estos a través de diversas herramientas tecnológicas (O'Flaherty y Phillips, 2015; Tourón y Santiago, 2015). Desde este enfoque, el personal docente 
http://doi.org/10.15359/ree.25-3.5

http://www.una.ac.cr/educare

educare@una.ac.cr

produce o selecciona el material digital que incluye los contenidos de la asignatura y actividades para verificar la comprensión de este material (Olaizola, 2015). En suma, el estudiantado puede acceder a la información compartida por su docente desde cualquier lugar y organizar sus tiempos de la forma que crean más conveniente para poder prepararse, con fin de demostrarlo en las actividades de evaluación propuestas por el personal docente.

Una de las recomendaciones para llevar a la práctica el enfoque pedagógico aprendizaje invertido es tener en cuenta, en primer lugar, las actividades que el estudiantado deben desarrollar fuera de aula, por ello es necesario seleccionar o producir el material digital, idear las actividades para asegurar la lectura y diagnosticar la comprensión del material digital para luego distribuirlo. En segundo lugar, hay que considerar las actividades que deben ejecutarse dentro del aula: la introducción, resolución de preguntas, discusión de dudas y su puesta en común, las actividades en el aula y el cierre de la sesión (Olaizola, 2015). Como se puede notar, se destacan dos fases imprescindibles durante la aplicación de este enfoque, el antes y el durante la clase presencial, cada una está compuesta de una serie de actividades planificadas cuyo principal objetivo es lograr mantener activo al estudiantado, fomentar su participación y convertirse en un medio para el logro de aprendizajes esperados.

En esta línea, en este enfoque pedagógico el estudiantado pasa de ser el receptor pasivo de información a diseñador y protagonista de su propio conocimiento (Chilingaryan y Zvereva, 2017), debido a que se centra en el estudiantado y específicamente en el desarrollo de sus competencias (Martín Rodríguez y Santiago Campión, 2016). El estudiantado es quien se responsabiliza de su propio aprendizaje (Bergmann y Sams, 2012), se tienen en cuenta sus necesidades individuales al disponerse de tiempo en aula para ello y se le brinda oportunidad de interactuar con sus pares (Vadillo Casero, 2018). La educación personalizada cobra gran importancia dentro de este enfoque, puesto que dar la vuelta a la clase "asegura que los alumnos reciben una educación personalizada, diseñada a la medida de sus necesidades individuales" (Bergmann y Sams, 2014, p. 19). Se toman en cuenta sus propios ritmos de estudio, al tener la posibilidad de ver el material digital las veces que consideren necesario (Bergmann y Sams, 2012; Heijstra y Sigurðardóttir, 2018; Sánchez Cruzado, 2017). El aprendizaje invertido promueve que el personal docente centre su atención en la formación de cada estudiante, al asegurar la comprensión individual del material digital compartido y al disponer de tiempo en la clase presencial para poder atender las dificultades que se presentarán al estudiantado durante su preparación fuera de aula.

Otro punto que hay que considerar de este enfoque es que permite el desarrollo del pensamiento crítico e independiente en el estudiantado, construyendo así la capacidad para el aprendizaje a lo largo de toda la vida (O'Flaherty y Phillips, 2015). El aprendizaje invertido impulsa el desarrollo de la mayor parte de los niveles de la taxonomía de Bloom; puesto que, fuera del aula, el estudiantado se ejercita en los procesos cognitivos de menor nivel, los que 
http://doi.org/10.15359/ree.25-3.5

implican conocer, comprender y aplicar; mientras que en la clase presencial tendrían que hacer lo propio con los procesos de mayor complejidad: análisis, evaluación y creación (Berenguer Albaladejo, 2016; Tourón et al., 2014).

Al disponer del tiempo fuera de aula para que el estudiantado revise los contenidos, se aprovecha, de mejor forma, el tiempo para desarrollar competencias en el aula, tales como el pensamiento crítico, el trabajo equipo, entre otros, que son imprescindibles para el desempeño del estudiantado en la vida laboral. En efecto, este enfoque pedagógico ha permitido el desarrollo de las competencias transversales en el estudiantado (Peña et al., 2017). Es conveniente señalar que esto se logrará, si el diseño de las actividades apunta claramente al desarrollo de las competencias que la asignatura tiene como objetivo lograr en el estudiantado.

La autonomía ha sido también una de las ventajas atribuidas a la clase invertida (Berenguer Albaladejo, 2016; Chilingaryan y Zvereva, 2017), pues permite que surja la necesidad en el estudiantado de involucrarse en su propio proceso de aprendizaje (García Gómez, 2016), y de tomar decisiones sobre qué, cuándo y cómo debe estudiar (Chilingaryan y Zvereva, 2017). Le brinda la oportunidad de asumir su propio criterio para seleccionar las estrategias de aprendizaje más apropiadas a sus características y una organización individual para el logro de los aprendizajes previstos.

La interacción con el estudiantado suele ser mayor al aplicarse el aprendizaje invertido. Se incrementa el tiempo de contacto entre el personal docente y el estudiantado, pues no se trata de estar en el aula para escuchar la clase magistral de su docente, sino para recibir orientación y corregir los errores con ayuda del personal docente (Chilingaryan y Zvereva, 2017). En definitiva, el aprendizaje invertido aporta a un proceso de enseñanza-aprendizaje en el que se impulsan las habilidades comunicativas, sobre todo en el aula, pues con este enfoque se seleccionan actividades que le permiten al estudiantado expresar sus ideas, discutirlas y corregirlas en un clima de colaboración.

Por lo que respecta al papel del personal docente dentro de este enfoque, hay que reconocer que es esencial, no se reduce a la exposición de los contenidos y a la revisión de exámenes. Además, se debe tener en cuenta que invertir el aprendizaje va más allá de la edición y distribución de contenidos multimedia (Tourón y Santiago, 2015). Desde la perspectiva del aprendizaje invertido, el personal docente se convierte en un facilitador que crea, guía, orienta y brinda retroalimentación durante las actividades de aprendizaje desarrolladas en el aula. El aprendizaje invertido "supone para él una mayor exigencia y profesionalidad y, por otro lado, su participación aporta un mayor valor añadido al aprendizaje" (Vadillo Casero, 2018, p. 159). La mayor exigencia se encuentra, quizás, en la dedicación de esfuerzos y tiempo para atender las necesidades de aprendizaje en el aula durante sus interacciones con el estudiantado; pues "el profesor, al liberarse del tiempo de clase que utiliza para la instrucción a los estudiantes, 
http://doi.org/10.15359/ree.25-3.5

http://www.una.ac.cr/educare

educare@una.ac.cr

puede centrarse en la atención de las necesidades puntuales de aprendizaje y dar respuesta a la diversidad y a los distintos ritmos de aprendizajes" (Fernández et al., 2017, p. 1122). Así, dentro de la evaluación formativa, el personal docente juega un papel central al monitorear e intervenir durante el proceso de aprendizaje, valiéndose de rúbricas u otros instrumentos de evaluación que permitan una retroalimentación acertada; igualmente tiene la tarea de estar pendiente de la revisión y retroalimentación de trabajos que se dejan de forma virtual, con el fin de verificar la preparación del estudiantado para la clase presencial.

En este punto, hay que reflexionar sobre la importancia de la disposición y preparación del personal docente en este enfoque, es vital que revise experiencias y entienda bien el enfoque, esto es crucial para así evitar el peligro de aplicar el aprendizaje invertido de forma inapropiada (O'Flaherty y Phillips, 2015). Durante la etapa de planificación se precisa de un personal docente preparado en el sentido del enfoque y en las diversas rutas de trasladarlo al aula, este proceso implica una reflexión profunda para la selección de las estrategias más apropiadas a la realidad de sus estudiantes. Es necesario anotar que se requiere para ello un personal docente motivado y convencido de la utilidad del enfoque (Aguilera-Ruiz et al., 2017), esto le permitirá introducir también al estudiantado al modelo, indicándole, de forma clara, cómo se llevará a cabo y mantenerlo motivado durante el curso (Berenguer Albaladejo, 2016).

La aplicación del aprendizaje invertido exige nuevas habilidades en el personal docente (Chilingaryan y Zvereva, 2017), como su manejo de los recursos y herramientas tecnológicas, pues debe seleccionar o producir el material digital, diseñar las actividades para asegurar la comprensión del material y distribuirlo (Olaizola, 2015). Además, es necesario que demuestre cierto grado de habilidades comunicativas (Aguilera-Ruiz et al., 2017).

Asimismo,

Es importante señalar que, aunque con este método pedagógico el alumno trabaja de forma autónoma, nunca lo hace solo porque el profesor actúa de guía en su proceso de aprendizaje, seleccionando los contenidos que debe estudiar, asimilar y retener, poniéndolos a su disposición a través de diversos medios y estando en constante comunicación con él. Lo único que implica es un cambio de roles respecto al modelo tradicional ya que el alumno debe colaborar activamente en su propio aprendizaje. (Berenguer Albaladejo, 2016, p. 1469)

En efecto, la labor del personal docente implica acompañamiento continuo al aprendizaje del estudiantado, no se reduce a la selección y difusión de recursos, sino que su rol le exige una mayor presencia fuera y dentro del aula, todo ello con el fin de orientar, motivar y facilitar experiencias de aprendizaje auténtico. 
http://doi.org/10.15359/ree.25-3.5

\section{Metodología}

Este estudio es de tipo descriptivo, cuasiexperimental y de enfoque mixto cuantitativocualitativo, debido a que el propósito fue identificar las actitudes del estudiantado acerca del aprendizaje invertido que experimentaron en el curso de Lenguaje y Comunicación. Consistió en la planificación y ejecución de sesiones siguiendo el enfoque de aprendizaje invertido, puesto que incluyeron actividades para los espacios individuales y grupales como característica fundamental. Al finalizar el curso, se aplicó un instrumento para el recojo de datos acerca de las actitudes del estudiantado.

El instrumento que se empleó fue un cuestionario compuesto por 10 enunciados organizados en una escala de Likert y cuatro preguntas abiertas ( $A, B, C$ y $D)$. El cuestionario fue elaborado por Farrah y Qawasmeh (2018), el mismo que pasó, como se detalla en el estudio, por un proceso de medición de confiabilidad y validez. El resultado del coeficiente de alfa de Cronbach fue de $(r=73)$, lo que indica un buen grado de consistencia interna $y$, por tanto, confiabilidad. Por otro lado, las personas autoras validaron el instrumento mediante la técnica de juicio experto. Se adecuó el cuestionario al tipo de participante que iba a responderlo, en este caso, al estudiantado, y se tradujo al español.

El instrumento se aplicó a 70 estudiantes de una universidad pública peruana que cursaron la asignatura de Lenguaje y Comunicación, en la cual se aplicó el enfoque aprendizaje invertido durante el semestre académico 2018-II. Se aplicó un muestreo no probabilístico por conveniencia (Hernández Sampieri et al., 2014), considerando al estudiantado matriculado en dicha asignatura durante el semestre indicado. La muestra está compuesta de 47 mujeres y 23 varones, cuyas edades fluctúan entre los 17 y 22 años.

Posteriormente, se analizaron los datos cuantitativos que se obtuvieron de la aplicación del cuestionario, para ello se realizó la tabulación de cada enunciado que contiene el instrumento utilizando la estadística descriptiva. Por otro lado, las respuestas obtenidas de las preguntas abiertas se analizaron de forma cualitativa, dichas respuestas permitieron hallar detalles acerca de los beneficios, diferencias en relación con el enfoque tradicional, dificultades y soluciones que percibe el estudiantado con respecto al aprendizaje invertido. De esta manera, complementaron los datos cuantitativos que se obtuvieron a partir de la escala de Likert.

\section{Resultados, análisis y discusión}

Los resultados del cuestionario aplicado se presentan, en la Tabla 1, de forma cuantitativa con base en las frecuencias, porcentajes obtenidos y media en cada ítem según los componentes de la escala de Likert. 
http://doi.org/10.15359/ree.25-3.5

http://www.una.ac.cr/educare

educare@una.ac.cr

Tabla 1: Frecuencias, porcentajes, medias y desviación estándar

\begin{tabular}{|c|c|c|c|c|c|c|c|c|}
\hline N. ${ }^{\circ}$ & Enunciados & $\begin{array}{l}\text { Totalmente en } \\
\text { desacuerdo }\end{array}$ & $\begin{array}{c}\text { En } \\
\text { desacuerdo }\end{array}$ & Neutral & $\begin{array}{c}\text { De } \\
\text { acuerdo }\end{array}$ & $\begin{array}{l}\text { Totalmente } \\
\text { de acuerdo }\end{array}$ & Media & DS \\
\hline \multirow[t]{2}{*}{1} & \multirow{2}{*}{$\begin{array}{l}\text { La clase invertida ayuda al estudiantado a } \\
\text { convertirse en aprendiz autónomo. }\end{array}$} & 0 & 0 & 6 & 26 & 38 & 4,46 & 0,65 \\
\hline & & $0 \%$ & $0 \%$ & $8,6 \%$ & $37,1 \%$ & $54,3 \%$ & & \\
\hline \multirow[t]{2}{*}{2} & \multirow{2}{*}{$\begin{array}{l}\text { La clase invertida permite al estudiantado tener } \\
\text { acceso a las presentaciones, lecturas, videos, etc. } \\
\text { en cualquier momento y de forma fácil. }\end{array}$} & 0 & 2 & 1 & 24 & 43 & 4,54 & 0,67 \\
\hline & & $0 \%$ & $2,9 \%$ & $1,4 \%$ & $34.3 \%$ & $61,4 \%$ & & \\
\hline \multirow[t]{2}{*}{3} & \multirow{2}{*}{$\begin{array}{l}\text { La clase invertida ayuda al estudiantado a } \\
\text { formular preguntas y obtener respuestas } \\
\text { inmediatas sobre los temas difíciles. }\end{array}$} & 0 & 1 & 16 & 32 & 21 & 4,04 & 0,77 \\
\hline & & $0 \%$ & $1,4 \%$ & $22,9 \%$ & $45,7 \%$ & $30 \%$ & & \\
\hline \multirow[t]{2}{*}{4} & \multirow{2}{*}{$\begin{array}{l}\text { La clase invertida da al estudiantado más } \\
\text { oportunidades para comunicarse entre sí. }\end{array}$} & 0 & 1 & 4 & 23 & 42 & 4,51 & 0,67 \\
\hline & & $0 \%$ & $1,4 \%$ & $5,7 \%$ & $32,9 \%$ & $60 \%$ & & \\
\hline \multirow[t]{2}{*}{5} & \multirow{2}{*}{$\begin{array}{l}\text { En la clase invertida, el personal docente está } \\
\text { disponible para una mayor interacción con sus } \\
\text { estudiantes. }\end{array}$} & 0 & 4 & 7 & 27 & 32 & 4,24 & 0,85 \\
\hline & & $0 \%$ & $5,7 \%$ & $10 \%$ & $38,6 \%$ & $45,7 \%$ & & \\
\hline \multirow[t]{2}{*}{6} & \multirow{2}{*}{$\begin{array}{l}\text { El estudiantado recomendaría la clase invertida } \\
\text { a sus amistades. }\end{array}$} & 1 & 0 & 14 & 28 & 27 & 4,14 & 0,83 \\
\hline & & $1.4 \%$ & $0 \%$ & $20 \%$ & $40 \%$ & $38,6 \%$ & & \\
\hline \multirow[t]{2}{*}{7} & \multirow{2}{*}{$\begin{array}{l}\text { La clase invertida reduce la cantidad de sesiones } \\
\text { frustrantes. }\end{array}$} & 0 & 4 & 11 & 29 & 25 & 4,09 & 0,87 \\
\hline & & $0 \%$ & $5,7 \%$ & $15,7 \%$ & $41,4 \%$ & $35,7 \%$ & & \\
\hline \multirow[t]{2}{*}{8} & \multirow{2}{*}{$\begin{array}{l}\text { La clase invertida permite al alumnado tener } \\
\text { más tiempo para la familia, amigos, amigas, } \\
\text { juegos y actividades extracurriculares. }\end{array}$} & 1 & 13 & 34 & 13 & 9 & 3,23 & 0,95 \\
\hline & & $1,4 \%$ & $18,6 \%$ & $48,6 \%$ & $18,6 \%$ & $12,9 \%$ & & \\
\hline \multirow[t]{2}{*}{9} & \multirow{2}{*}{$\begin{array}{l}\text { El estudiantado prefiere ver a un sujeto docente } \\
\text { que desarrolla una lección tradicional que una } \\
\text { lección en la que emplea herramientas digitales. }\end{array}$} & 19 & 30 & 17 & 4 & 0 & 2,09 & 0,86 \\
\hline & & $27,1 \%$ & $42,9 \%$ & $24,3 \%$ & $5,7 \%$ & $0 \%$ & & \\
\hline \multirow[t]{2}{*}{10} & \multirow{2}{*}{$\begin{array}{l}\text { La clase invertida ha mejorado el aprendizaje } \\
\text { del estudiantado. }\end{array}$} & 0 & 0 & 9 & 38 & 23 & 4,20 & 0,65 \\
\hline & & $0 \%$ & $0 \%$ & $12,9 \%$ & $54,3 \%$ & $32,9 \%$ & & \\
\hline
\end{tabular}

Nota: Elaboración propia.

Se identificó el significado de los promedios con base en los siguientes intervalos propuestos por Farrah y Qawasmeh (2018): 1 a 1,79 totalmente en desacuerdo; 1,8 a 2,59 en desacuerdo; 2,6 a 3,39 neutral; 3,4 a 4,19 de acuerdo; $y, 4,2$ a 5 totalmente de acuerdo, Los resultados muestran que los promedios más altos corresponden a los ítems 1, 2, 4, 5 y 10; lo cual evidencia que el estudiantado en su mayoría se encuentra totalmente de acuerdo en que el aprendizaje invertido ayuda a desarrollar la autonomía, les permite tener acceso a los recursos, brinda oportunidades de comunicarse entre sí y con su docente; y, que ha mejorado sus aprendizajes. 
http://doi.org/10.15359/ree.25-3.5

Los resultados también indican que el estudiantado se muestra en desacuerdo con respecto a un personal docente que desarrolle una clase tradicional, pues sus preferencias se orientan a un personal docente que use herramientas tecnológicas para compartir contenido como sucede en el aprendizaje invertido. Asimismo, están de acuerdo en que el aprendizaje invertido reduce las sesiones frustrantes, les ayuda a formular preguntas y obtener respuestas de forma inmediata y lo recomendarían. Esta información evidencia sus actitudes positivas hacia este enfoque.

Además, es necesario observar que el estudiantado se muestra neutral en que el aprendizaje invertido les permita tener tiempo para familia amigos u otras actividades. Este resultado posiblemente se deba a que, al tener actividades de preparación en espacios individuales fuera de aula, este enfoque requiere dedicar tiempo considerable en la revisión y análisis de los recursos didácticos, además del desarrollo de una actividad que evidencie este análisis.

Con respecto a las preguntas abiertas, estas se orientaron también a conocer las actitudes del estudiantado sobre el enfoque aprendizaje invertido luego de haber participado en esta experiencia durante la asignatura, se analizó cada una de las respuestas recibidas. Las conclusiones se presentan a continuación.

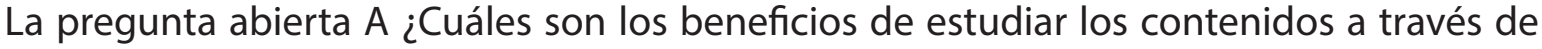
herramientas digitales antes de asistir a clase presencial? El estudiantado señaló que entre los principales beneficios de este enfoque es que perciben una mayor facilidad y flexibilidad para revisar los materiales, pueden repasar con detenimiento, elegir sus propios horarios de estudio y elegir el ambiente más adecuado para estudiar:

Participante 30: Nos da un mayor y más fácil acceso a las lecturas, tareas, etc.

Participante 16: Que puedo acceder a los recursos en cualquier momento.

Participante 4: Me da más tiempo de análisis del tema y verlo en cualquier lugar.

Participante 45: Tener mayor cantidad de tiempo para interiorizar y criticar el contenido.

Asimismo, por el hecho de tener a disposición todos los contenidos pueden poseer más tiempo para analizar la información, interiorizar y comparar ideas al buscar otras fuentes. Consideran que todo ello les permite explorar sus conocimientos previos, obtener más preparación y dominar los temas propuestos, lo que les da mayor seguridad para opinar en aula y participar en las actividades presenciales: 
http://doi.org/10.15359/ree.25-3.5

http://www.una.ac.cr/educare

educare@una.ac.cr

Participante 66: Nos permite estar preparados y tener noción del tema que se tocará, por ello, facilita el aprendizaje.

Participante 53: Permite tener conocimientos previos; y, en clase, enfocarse en las dudas.

Participante 69: Permite que el estudiante indague más del tema a tratar para estar preparado ante un posible debate en clase.

Participante 23: Nos prepara para una mayor interacción en clase.

Acerca de la pregunta abierta B ¿qué diferencias, si las hay, observa entre el rol del personal docente en la clase invertida con el rol del personal docente en una clase tradicional?, el estudiantado coincide en señalar que la diferencia es clara entre estos dos roles. La mayoría destaca que en el enfoque aprendizaje invertido, el personal docente se convierte en facilitador y propicia mayor participación del estudiantado; percibe que hay mayor interacción entre el estudiantado y entre docente-estudiante que en una clase tradicional en la que el personal docente solo expone el tema. El estudiantado, además de lo mencionado, destacó que, en el aprendizaje inverso, el personal docente impulsa el desarrollo de habilidades como la comunicación efectiva y el trabajo en equipo. Por último, perciben que el personal docente tiene una atención más personalizada en el aprendizaje invertido que en el modelo tradicional.

Participante 57: En la clase tradicional, los alumnos no intercambian opiniones, lo cual sí ocurre en la clase invertida. En la clase invertida, se trabajan habilidades blandas y es más personalizado.

Participante 60: La clase invertida hace que el alumno sea el protagonista, que tenga un mayor desenvolvimiento, ya que sería el que brinde y exprese sus ideas haciendo que tenga más seguridad.

Participante 28: El profesor actúa como facilitador o guía en la clase invertida.

Participante 26: La clase invertida permite el desarrollo de habilidades como la comunicación efectiva.

Participante 68: En la clase invertida, hay más participación en equipos.

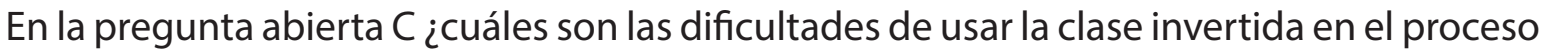
de aprendizaje?, el estudiantado respondió que una de las mayores dificultades es que algunos sujetos pueden no sentirse verdaderamente comprometidos con su aprendizaje, es decir, que no se organicen y no revisen previamente el material brindado, por lo tanto, no se encuentren 
http://doi.org/10.15359/ree.25-3.5

preparados para participar en el aula. Otra de las dificultades es que no todas las personas tienen acceso a internet para poder estudiar con facilidad y en algunos casos también indicaron que hay algún estudiantado que no cuenta con los dispositivos para acceder al material digital ya sea laptop, tabletas o celulares, lo cual significa una limitación. También se pudo observar, en sus respuestas, que las dudas que pueden tener durante su preparación fuera de aula pueden no ser resueltas si el estudiantado es tímido y no se atreve a contactarse con su docente.

Participante 49: Algunas veces, hay alumnos que no pudieron realizar sus trabajos previos.

Participante 45: La falta de compromiso de los estudiantes.

Participante 39: No siempre todos los estudiantes tienen la posibilidad de poseer algún aparato digital como celular o laptop.

Participante 18: No tener Internet o algún aparato tecnológico.

Participante 3: Hay estudiantes que presentan timidez para expresarse.

Participante 35: Las dudas que puedan tener los estudiantes, antes de la clase presencial, tienen temor de preguntar al docente incluso por el medio virtual.

Para la pregunta abierta $\mathrm{D}$ ¿cuáles son las soluciones para las dificultades que enfrenta el estudiantado durante la clase invertida?, el estudiantado planteó que deben sentirse más comprometidos para poder planificar y organizar sus tiempos de modo que pueden prepararse de forma autónoma al analizar el material con anticipación y desarrollar las actividades propuestas por el personal docente. Asimismo, indican que sería provechoso un recurso digital que le permita acceder al material de clase sin necesidad de estar conectado a internet e incorporar alguna estrategia virtual para mantenerse en contacto directo y permanente con su docente.

Participante 1: Tener más medios de contacto con el profesor para resolver las dudas.

Participante 23: Compromiso estudiantil.

Participante 54: Una aplicación que no necesite Internet para visualizar los archivos de la clase.

Participante 65: Que el estudiante sea más autónomo en la búsqueda de conocimiento.

Participante 70: Más responsabilidad de parte de los estudiantes. 
http://doi.org/10.15359/ree.25-3.5

http://www.una.ac.cr/educare

educare@una.ac.cr

El propósito del presente estudio fue determinar las actitudes de una muestra del estudiantado de una universidad pública sobre el aprendizaje invertido. Se aplicó un cuestionario de escala Likert para que expresaran su nivel de acuerdo o desacuerdo en razón a 10 reactivos; además, el instrumento incluyó cuatro preguntas abiertas para obtener información más detallada sobre dichas actitudes. En los resultados se puede observar que hubo mayores porcentajes de acuerdo y totalmente de acuerdo en 8 de los 10 reactivos del cuestionario, lo cual indica que gran parte del estudiantado tiene actitudes favorables hacia el aprendizaje invertido, pues identifica las ventajas que tiene. Sumado a ello, luego del análisis de las respuestas a las preguntas abiertas se refuerzan estos resultados, puesto que el estudiantado destaca los beneficios que tiene el aprendizaje invertido para un mejor aprovechamiento de los espacios grupales y el rol protagónico que se le asigna al estudiantado en su propio proceso de aprendizaje.

Estos resultados coinciden con el estudio realizado por Arráez Vera et al. (2018), quienes en su estudio llegaron a la conclusión de que un porcentaje muy alto de estudiantes prefiere la clase invertida a la clase tradicional (77,7\%). Del mismo modo, los resultados también advierten que el $40 \%$ del estudiantado reconoce no dedicar tiempo suficiente a revisar el material compartido fuera de aula, lo cual también fue advertido en las respuestas del estudiantado en la presente investigación, pues identifican que mucho estudiantado no asume verdaderamente la responsabilidad de prepararse fuera del aula, lo cual significa una dificultad para alcanzar los logros esperados.

Por otro lado, los resultados de este estudio también se encuentran en consonancia con los de Fauzan y Novary Ngabut (2018), quienes, en sus conclusiones, afirman que la mayoría del estudiantado percibió que el enfoque flipped learning o aprendizaje invertido fue una experiencia beneficiosa, también se llegó a la conclusión de que se implementa de forma efectiva siempre y cuando el estudiantado tenga el compromiso de aprender.

Finalmente, se observa similitud de los resultados de esta investigación con los de Farrah y Qawasmeh (2018), pues en estos últimos el estudiantado muestra actitudes favorables al aprendizaje invertido, puesto que se involucra y participa de forma colaborativa e interactiva con sus pares y docentes, para la mayoría el aprendizaje invertido potencia sus habilidades comunicativas y reduce las sesiones frustrantes que tenían antes.

Este tipo de estudios son fundamentales para el personal docente, porque permiten obtener información acerca de cómo perciben los actores principales la implementación del aprendizaje invertido. Es necesario identificar no solo cuánto conoce de este mismo, sino también cuáles son los principales obstáculos y cómo considera que pueden solucionarse. Esta información aportará incluso a la mejora del desempeño docente, puesto que el personal sabrá si las acciones de mejora deben enfocarse en los espacios individuales o grupales. 


\section{Conclusiones}

Las actitudes del estudiantado sobre el enfoque aprendizaje invertido son positivas en la medida en que reconocen una serie de beneficios para el logro de sus aprendizajes en el curso de Lenguaje y Comunicación. Entre los principales beneficios que más destacan es que le permite sentirse activo durante el proceso de enseñanza-aprendizaje y fortalecen su autonomía al asumir la responsabilidad de prepararse fuera del aula. Asimismo, reconoce que el tiempo se aprovecha mejor en el aula, puesto que le brinda la oportunidad de desenvolverse y de comunicarse mejor con su docente y con sus pares. La mayoría del estudiantado prefiere una persona docente facilitadora de experiencias significativas de aprendizaje que una tradicional o mera expositora de contenidos.

Los principales desafíos que el estudiantado identifica con respecto a este enfoque pedagógico principalmente se vinculan a las dificultades de acceso a internet para quienes que no cuentan con recursos para hacerlo. Por otro lado, la posible falta de interés de algún estudiantado para prepararse de forma autónoma fuera de aula.

El aprendizaje invertido exige al personal docente un dominio teórico y práctico del enfoque para su correcta implementación: elaboración y envío de material educativo, las actividades de preparación y la retroalimentación en los espacios individuales fuera de aula con ayuda de las herramientas digitales; por otro lado, el diseño y ejecución de actividades en los espacios grupales en aula que le permitan al estudiantado interactuar con sus pares y docentes, profundizar en los contenidos y desarrollar procesos cognitivos de orden superior. Además, es necesario que el personal docente motive y explique, de forma detenida, al estudiantado en qué consiste y cuál es la relevancia del enfoque, de tal forma que este comprenda la dinámica de trabajo y se comprometa a participar de forma activa y autónoma en las actividades programadas.

Resulta relevante realizar estetipo de estudios que identifiquen lasactitudes del estudiantado, porque brinda información que puede resultar útil para la mejora del desempeño docente de quienes aplican el aprendizaje invertido en sus cursos. Es una oportunidad de reflexión docente para identificar las principales dificultades que pueden suscitarse durante su implementación desde las perspectivas del estudiantado y que tienen que ver, directamente, con el rol del personal docente, pues esta información servirá para plantear las acciones correctivas necesarias.

Asimismo, es recomendable continuar esta línea de investigación con otros estudios que trabajen con muestras mayores y que permitan identificar qué otras dificultades se presentan en la implementación del aprendizaje invertido no solo desde la perspectiva del estudiantado, sino también del personal docente. Otra línea interesante que puede resultar muy productiva es analizar las características del proceso de evaluación formativa que supone el aprendizaje invertido. Es oportuno determinar los protocolos y mecanismos de la retroalimentación, sobre todo en los espacios individuales de preparación, de modo que se acompañe al estudiantado en su proceso de aprendizaje también fuera de aula. 
http://doi.org/10.15359/ree.25-3.5

http://www.una.ac.cr/educare

educare@una.ac.cr

\section{Declaración de Material complementario}

Este artículo tiene disponible, como material complementario:

-La versión preprint del artículo en https://doi.org/10.5281/zenodo.4041936

\section{Referencias}

Aguilera-Ruiz, C., Manzano-León, A., Martínez-Moreno, I., Lozano-Segura, M. del C. y Casiano Yanicelli, C. (2017). El modelo Flipped Classroom. Revista INFAD de Psicología, 4(1), 261266. https://doi.org/10.17060/ijodaep.2017.n1.v4.1055

Akçayır, G. y Akçayır, M. (2018). The flipped classroom: A review of its advantages and challenges. Computers and Education, 126, 334-345. https://doi.org/10.1016/j.compedu.2018.07.021

Arráez Vera, G., Lorenzo Lledó, A., Gómez Puerta, M. y Lorenzo Lledó, G. (2018). La clase invertida en la educación superior: Percepciones del alumnado. International Journal of Developmental and Educational Psychology, 2(1), 155-162. https://doi.org/10.17060/ ijodaep.2018.n1.v2.1197

Awidi, I. T. y Paynter, M. (2019). The impact of a flipped classroom approach on student learning experience. Computers \& Education, 128, 269-283. https://doi.org/10.1016/j. compedu.2018.09.013

Berenguer Albaladejo, C. (2016). Acerca de la utilidad del aula invertida o flipped classroom. En M. T. Tortosa Ybáñez, S. Grau Company y J. D. Álvarez Teruel (Coord.), XIV Jornadas de Redes de Investigación en Docencia Universitaria. Investigación, innovación y enseñanza universitaria: Enfoques pluridisciplinares (pp. 1466-1480). Universidad de Alicante. http:// rua.ua.es/dspace/handle/10045/59358

Bergmann, J. y Sams, A. (2012). Flip your classroom: Reach every student in every class every day. International Society for Technology in Education. http://i-lib.imu.edu.my/NewPortal/ images/NewPortal/CompE-Books/Flip-Your-Classroom.pdf

Bergmann, J. y Sams, A. (2014). Dale la vuelta a tu clase: Lleva tu clase a cada estudiante, en cualquier momento y cualquier lugar. Ediciones SM.

Chilingaryan, K. y Zvereva, E. (2017). Methodology of flipped classroom as a learning technology in foreign language teaching. Procedia - Social and Behavioral Sciences, 237, 1500-1504. https://doi.org/10.1016/J.SBSPRO.2017.02.236

Farrah, M. y Qawasmeh, A. (2018). English students' attitudes towards using flipped classrooms in language learning at Hebron University. Research in English Language Pedagogy RELP, 6(2), 275-294. http://relp.khuisf.ac.ir/article 542708 ad8efd7e2a1d65aaf5a0851191717c9a.pdf 
http://doi.org/10.15359/ree.25-3.5

Fauzan, A. y Novary Ngabut, M. (2018). EFL students' perception on flipped learning in writing class. Journal on English as a Foreign Language, 8(2), 115-129. https://doi. org/10.23971/jefl.v8i2.792

Fernández, M., Godoy Guglielmone, M. V., Mariño, S. I. y Barrios, W. (2017) ¿Invirtiendo la clase o invirtiendo en la clase? Enseñanza de tecnología para la producción multimedia mediante aula invertida. 23 Congreso Argentino de Ciencias de la Computación. RedUNCI. http://sedici. unlp.edu.ar/handle/10915/63889

García Gómez, A. (2016). Aprendizaje inverso y motivación en el aula universitaria. Pulso: Revista de Educación, 39, 199-218. https://dialnet.unirioja.es/servlet/articulo?codigo=5823495

Heijstra, T. M. y Sigurðardóttir, M. S. (2018). The flipped classroom: Does viewing the recordings matter? Active Learning in Higher Education, 19(3), 211-223. https://doi. org/10.1177/1469787417723217

Hernández Sampieri, R., Fernández Collado, C. y Baptista Lucio, P. (2014). Metodología de la investigación (6. ${ }^{\mathrm{a}}$ ed.). McGraw-Hill.

López, M. y Flores Herrera, J. R. (2013). La clase invertida para fomentar la conceptualización. Revista Científica YACHANA, 2(2), 77-81. http://revistas.ulvr.edu.ec/index.php/yachana/ article/viewFile/40/35

Martín Rodríguez, D. y Santiago Campión, R. (2016). Flipped Learning en la formación del profesorado de secundaria y bachillerato. Formación para el cambio. Contextos Educativos Revista de Educación, 1, 117-134. https://doi.org/10.18172/con.2854

Melo, L. y Sánchez, R. (2017). Análisis de las percepciones de los alumnos sobre la metodología flipped classroom para la enseñanza de técnicas avanzadas en laboratorios de análisis de residuos de medicamentos veterinarios y contaminantes. Educación Química, 28(1), 30-37. https://doi.org/10.1016/j.eq.2016.09.010

O'Flaherty, J. y Phillips, C. (2015). The use of flipped classrooms in higher education: A scoping review. The Internet and Higher Education, 25, 85-95. https://doi.org/10.1016/j. iheduc.2015.02.002

Olaizola, A. (2015). La clase invertida:Una experiencia en la materia introducción a la investigación. Revista electrónica de didáctica en Educación Superior, 10, 1-14. http://ojs.cbc.uba.ar/index. $\mathrm{php/redes/article/view/82}$

Peña, B., Zabalza, I., Usón, S., Llera, E. M., Martínez, A. y Romeo, L. M. (julio, 2017). Experiencia piloto de aula invertida para mejorar el proceso de enseñanza-aprendizaje en la asignatura de Termodinámica Técnica. Trabajo presentado en el III Congreso Nacional de Innovación Educativa y Docencia en Red. Universidad Politécnica de Valencia, España. http://dx.doi. org/10.4995/INRED2017.2017.6868 
http://doi.org/10.15359/ree.25-3.5

http://www.una.ac.cr/educare

educare@una.ac.cr

Sánchez Cruzado, C. (2017). Flipped classroom. La clase invertida, una realidad en la Facultad de Ciencias de la Educación de la Universidad de Málaga [Tesis doctoral]. Universidad de Málaga, España. https://riuma.uma.es/xmlui/handle/10630/14993

Sánchez Vera, M. del M., Solano Fernández. I. M. y Gonzalez Calatayud, V. (2016). FLIPPED-TIC: Una experiencia de Flipped Classroom con alumnos de Magisterio / FLIPPED-TIC: A Flipped Classroom experience with preservice teachers. Revista Latinoamericana de Tecnología Educativa-RELATEC, 15(3), 69-81. https://doi.org/10.17398/1695-288X.15.3.69

Tourón, J. y Santiago, R. (2015). El modelo Flipped Learning y el desarrollo del talento en la escuela. Revista de Educación, 368, 196-231. 10.4438/1988-592X-RE-2015-368-288

Tourón, J., Santiago, R. y Díez, A. (2014). The Flipped Classroom: Cómo convertir la escuela en un espacio de aprendizaje. Grupo Océano. http://www.digital-text.com/wp-content/ uploads/2015/03/FlippedClassroom.pdf

Vadillo Casero, P. J. (2018). La clase invertida ("Flipped Classroom"), una nueva forma de aprender. Publicaciones Didácticas, Revista Profesional de Investigación, Docenciay Recursos Didácticos, 91, 145-164. https://publicacionesdidacticas.com/hemeroteca/pd_091_feb.pdf 